\title{
Internal strain monitoring in composite materials with embedded photonic crystal fiber Bragg gratings
}

Geernaert, Thomas, Sulejmani, Sanne, Sonnenfeld, Camille, Chah, Karima, Luyckx, Geert, et al.

Thomas Geernaert, Sanne Sulejmani, Camille Sonnenfeld, Karima Chah, Geert Luyckx, Nicolas Lammens, Eli Voet, Martin Becker, Hugo Thienpont, Francis Berghmans, "Internal strain monitoring in composite materials with embedded photonic crystal fiber Bragg gratings," Proc. SPIE 9226,

Nanophotonics and Macrophotonics for Space Environments VIII, 92260D (17 September 2014); doi: 10.1117/12.2061608

Event: SPIE Optical Engineering + Applications, 2014, San Diego, California, United States 


\title{
Internal strain monitoring in composite materials with embedded photonic crystal fiber Bragg gratings
}

\author{
Thomas Geernaert*a, Sanne Sulejmani ${ }^{\mathrm{a}}$, Camille Sonnenfeld ${ }^{\mathrm{a}}$, Karima Chah ${ }^{\mathrm{b}}$, Geert Luyckx $^{\mathrm{c}}$, \\ Nicolas Lammens ${ }^{\mathrm{c}}$, Eli Voet ${ }^{\mathrm{c}}$, Martin Becker ${ }^{\mathrm{d}}$, Hugo Thienpont ${ }^{\mathrm{a}}$, Francis Berghmans ${ }^{\mathrm{a}}$ \\ ${ }^{a}$ Vrije Universiteit Brussel, Department of Applied Physics and Photonics, Brussels Photonics Team, \\ Pleinlaan 2, 1050 Brussels, Belgium; ${ }^{\mathrm{b}}$ Université de Mons, Department of Electromagnetism and \\ Telecommunications, Boulevard Dolez 31, 7000 Mons, Belgium; 'Universiteit Gent, Department of \\ Materials Science and Engineering, Technologiepark 903, 9052 Gent, Belgium; ${ }^{d}$ Institute of \\ Photonic Technology, Albert-Einstein-Straße 9, 07745 Jena, Germany
}

\begin{abstract}
The possibility of embedding optical fiber sensors inside carbon fiber reinforced polymer (CFRP) for structural health monitoring purposes has already been demonstrated previously. So far however, these sensors only allowed axial strain measurements because of their low sensitivity for strain in the direction perpendicular to the optical fiber's axis. The design flexibility provided by novel photonic crystal fiber (PCF) technology now allows developing dedicated fibers with substantially enhanced sensitivity to such transverse loads. We exploited that flexibility and we developed a PCF that, when equipped with a fiber Bragg grating (FBG), leads to a sensor that allows measuring transverse strains in reinforced composite materials, with an order of magnitude increase of the sensitivity over the state-of-the-art. In addition it allows shear strain sensing in adhesive bonds, which are used in composite repair patches. This is confirmed both with experiments and finite element simulations on such fibers embedded in CFRP coupons and adhesive bonds. Our sensor brings the achievable transverse strain measurement resolution close to a target value of $1 \mu$ strain and could therefore play an important role for multi-dimensional strain sensing, not only in the domain of structural health monitoring, but also in the field of composite material production monitoring. Our results thereby illustrate the added value that PCFs have to offer for internal strain measurements inside composite materials and structures.
\end{abstract}

Keywords: photonic crystal fiber, microstructured optical fiber, optical fiber sensor, structural health monitoring, strain sensing, fiber Bragg grating, composite materials

\section{INTRODUCTION}

Photonic crystal fiber Bragg grating (PCFBG) sensors are becoming increasingly popular owing to the peculiar characteristics of photonic crystal fibers (PCFs) or microstructured optical fibers that cannot be achieved using conventional optical fiber technology. More specifically, the design flexibility of such PCFs allows developing sensors that exhibit selective sensitivities to e.g. axial strain, transverse strain or even shear stress, whilst being negligibly crosssensitive to temperature changes. This is a great asset for structural health monitoring (SHM) applications. Section 1 of this paper will briefly summarize the state-of-the-art on the use of PCF based sensors for SHM purposes.

Section 2 focuses on a specific PCF design, to which we refer as 'Butterfly' PCF. This fiber is highly birefringent and encodes transverse strain into the spectral distance $\Delta \lambda$ between the two Bragg peaks reflected by a fiber Bragg grating (FBG) fabricated in this fiber (Figure 1). Since the birefringence of that PCF is almost insensitive to temperature changes, the transverse strain measurement is close to being independent of temperature variations. In Section 3 we show that by using this PCF, we can simultaneously measure the normal strain in 3 dimensions inside a composite - the axial strain, the transverse in-plane strain and the transverse out-of-plane strain - with $\mu$ strain resolutions using commercially available FBG interrogator equipment. The transverse strain sensitivity proves also to be valuable in composite manufacturing monitoring. Finally, Section 4 deals with the potential of our PCFBG sensor for shear stress measurements and disbond monitoring in lap joints.

*tgeernae@vub.ac.be; phone +32 2629 1381; fax +32 2 629 3450; www.b-phot.org 


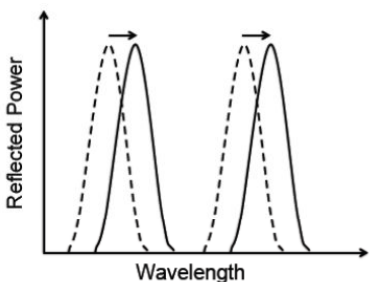

(a)

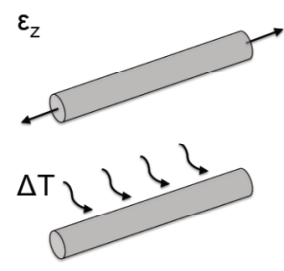

(b)

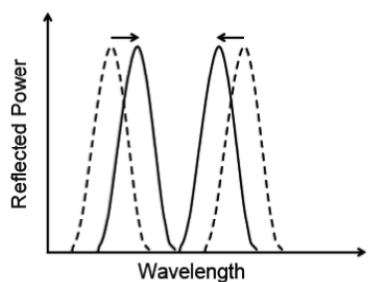

(c)

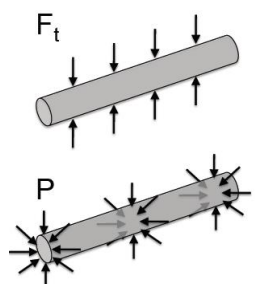

(d)

Figure 1: Differential sensing principle of the Butterfly PCFBG. Load applied transversally to the fiber is encoded in the spacing $\Delta \lambda$ between the two Bragg peaks. $(\mathrm{a}, \mathrm{b})$ Response of the sensor to temperature changes or axial strain - both Bragg peaks move in to higher or lower wavelengths and $\Delta \lambda$ remains unchanged. (c,d) Response of the sensor to transverse load - the Bragg peaks move in opposite direction and $\Delta \lambda$ changes.

\section{STATE-OF-THE-ART OF PCF FOR SHM-RELATED APPLICATIONS}

As stated above, PCF technology allows achieving optical fibers with sensing characteristics that cannot be obtained with conventional fiber technology. PCF sensing approaches and techniques have been reviewed for example by Frazao et al. [1], Canning [2] and Pinto et al. [3]. With respect to SHM related applications, PCF based sensors have been mostly combined with gratings inscribed in these fibers to enable measurements of mechanical quantities. A far from exhaustive list of examples includes pressure, transverse force, strain and bend sensing, with emphasis on avoiding strain and temperature cross-sensitivity or discriminating between strain and temperature effects [4]-[13]. Grating inscription techniques in PCF have been reviewed recently in [14].

\section{THE BUTTERFLY PCF AND GRATINGS WRITTEN THEREIN}

We have obtained the cross-section of our highly birefringent Butterfly PCF following extensive modeling that considered both the optical and the mechanical characteristics of the fiber [15]. We have modeled the sensitivity of the phase modal birefringence $B$ to external thermo-mechanical perturbations with the commercially available COMSOL Multiphysics ${ }^{\circledR}$ software [16].

The phase modal birefringence $B$ is defined as:

$$
B=\frac{\lambda}{2 \pi}\left(\beta_{x}-\beta_{y}\right)
$$

where $\beta_{\mathrm{x}}$ and $\beta_{\mathrm{y}}$ are the propagation constants of the orthogonally polarized fundamental modes propagating through the PCF. When fabricating a FBG with a period $\Lambda$ in a birefringent PCF, the grating will return two reflection peaks with a wavelength separation $\Delta \lambda$ (see also Figure 1) given by:

$$
\Delta \lambda=2 B \Lambda
$$

The polarimetric pressure and temperature sensitivities $K_{p}$ and $K_{T}$ are defined as ( $\alpha_{\mathrm{T}}$ is the linear thermal expansion coefficient):

$$
K_{p} \triangleq \frac{2 \pi}{\lambda} \frac{d B}{d p} ; K_{T} \triangleq \frac{2 \pi}{\lambda}\left(\frac{d B}{d T}+B \alpha_{T}\right)
$$

If we want to obtain a PCF that is very sensitive to pressure whilst being - in as much as possible - insensitive to temperature changes, the figure-of-merit $K_{p} / K_{T}$ should be maximized. To do so one has to be able to calculate $K_{p}$ and $K_{T}$. A full description of how this can be done is out of the scope of this paper. We refer to [15], [17] and [18] for a more detailed discussion.

The "Butterfly" PCF that we have obtained, with an ideal and as-built cross-section as shown in Figure 2, fulfills our requirements in terms of sensitivity, fiber manufacturability and optical guiding properties. 


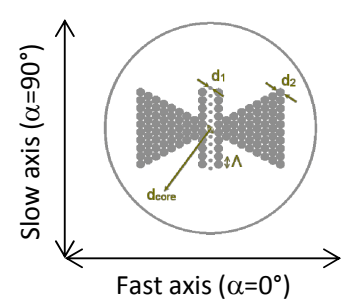

(a)

$\begin{array}{cc}\text { Parameter } & \text { Typical Value } \\ \text { Outer cladding diameter } & 125 \mu \mathrm{m} \\ \text { Number of rings of airholes } & 6-9 \\ \text { Airhole pitch } \Lambda & 3.5-4 \mu \mathrm{m} \\ \mathrm{d}_{1} / \Lambda & 0.225 \\ \mathrm{~d}_{2} / \Lambda & 0.95-0.99 \\ \mathrm{~d}_{\text {core }} / \Lambda & 0.9 \\ \text { Core doping concentration } & \sim 2.88 \mathrm{~mol} \%\end{array}$

(b)

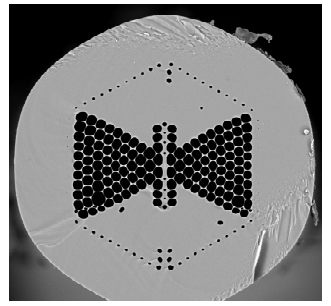

(c)

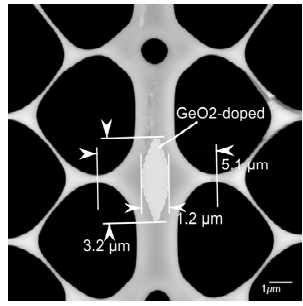

(d)

Figure 2: (a) Ideal cross-section of the Butterfly PCF (b) Ideal parameters of the butterfly PCF (c) Scanning Electron

Microscope photograph of the cross-section of the fabricated Butterfly PCF. (d) Close-up of the core region of the PCF.

\section{THREE-DIMENSIONAL STRAIN MEASUREMENTS IN COMPOSITE MATERIALS}

\section{Parallel embedded Butterfly PCFBGs}

Due to its particular structure, the sensitivity of the butterfly PCFBG to transverse strain varies in a sinusoidal manner with the angular orientation $\alpha$ of the microstructure with respect to the direction of the applied load. It is most sensitive when the load is applied along $\alpha=90^{\circ}$, which corresponds to the direction of the slow-axis indicated in Figure 2(a) [19]. This dependence of the sensitivity on the angular orientation can be exploited to quantify the strain along the 3 directions of space inside a carbon fiber reinforced polymer (CFRP) composite material. To do so, one needs to relate the normal strains in the 3 directions of space in the composite material to the measured changes in the 4 Bragg wavelengths returned by two closely spaced parallel PCFBG sensors embedded in a composite coupon with different angular orientations, as schematically illustrated in Figure 3.

The relation between the strains and the temperature in the material at the location of the sensors and the Bragg wavelength shifts is given by Equation (4).

$$
\left(\begin{array}{c}
\varepsilon_{1}^{h} \\
\varepsilon_{2}^{h} \\
\varepsilon_{3}^{h} \\
\Delta T
\end{array}\right)=[T C] \cdot[K]^{-1} \cdot\left(\begin{array}{l}
\Delta \lambda_{B 1,1 \prime} / \lambda_{B 1,1 \prime} \\
\Delta \lambda_{B 1,2 \prime} / \lambda_{B 1,2 \prime} \\
\Delta \lambda_{B 2,1 \prime} / \lambda_{B 2,1 \prime} \\
\Delta \lambda_{B 2,2 \prime} / \lambda_{B 2,2 \prime}
\end{array}\right)
$$

This equation uses the Transfer Coefficient matrix [TC] that relates the temperature and strain distributions in the host composite material to those in the optical fiber sensor in the coordinate system $(1 \mathrm{~h}, 2 \mathrm{~h}, 3 \mathrm{~h})$ of the host composite material [20], [21]. More specifically, $\varepsilon_{1}^{\mathrm{h}}$ is the axial strain, $\varepsilon_{2}^{\mathrm{h}}$ is the transverse in-plane strain and $\varepsilon_{3}^{\mathrm{h}}$ is the transverse out-ofplane strain (see also Figure 3). We obtained the [TC] matrix using extensive finite-element modelling. The sensitivity matrix $[\mathrm{K}]$ relates the temperature and strain changes in the optical fiber core to the output of the optical signal [22], [23]. This sensitivity matrix is obtained in an analytical form. $\lambda_{\mathrm{B} 1,1}$, and $\lambda_{\mathrm{B} 1,2}$, denote the initial unstrained mean Bragg wavelengths of the first PCFBG, and $\lambda_{\mathrm{B} 2,1}$, and $\lambda_{\mathrm{B} 2,2}$, are the initial unstrained mean Bragg wavelengths of the second PCFBG. $\Delta \lambda \mathrm{B}_{1,1}, \Delta \lambda_{\mathrm{B} 1,2^{\prime}}, \Delta \lambda_{\mathrm{B} 2,1}$, and $\Delta \lambda_{\mathrm{B} 2,2^{2}}$ are the shifts of these Bragg wavelength peaks that stem from thermomechanical load applied to the composite material. The coordinates 1' and 2' associated with the two Bragg wavelengths returned by one PCFBG correspond to the directions of the slow and fast axes of the PCF, respectively (see Figure 2).

Figure 4 (b) shows a typical result obtained for a transverse out-of-plane load applied to a CFRP coupon as illustrated in Figure 4 (a). We obtain a good agreement between our finite element modelling results and the strains measured and derived using Equation (4). The calculated axial strain in the composite material shows a relatively larger relative error of up to $50 \%$ when compared to the experimental data of the strain gauge. However, and since the axial strains are very low, this corresponds to a difference of only $24 \mu \varepsilon$ in the PCFBG configuration $0^{\circ} / 90^{\circ}$ as sketched in Figure 3 . The values of the transverse in-plane strain also exhibit some differences with respect to the measured strains, which can be explained by their low values. This stems from the fact that the expected strains along the $1 \mathrm{~h}$ - and $2 \mathrm{~h}$-directions have the same magnitude as the strain measurement resolutions of about $5 \mu \varepsilon$ (these resolutions are not discussed in this paper). The calculated transverse out-of-the plane strains exhibit an excellent agreement with the measured strains, with a relative error around $10 \%$. 


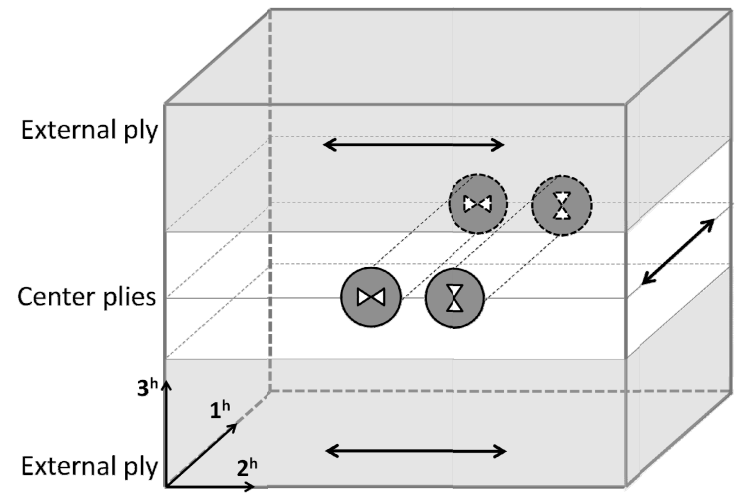

Figure 3: Illustration of 2 closely space PCFBGs embedded in the middle of a laminate composite (not drawn to scale) with the longitudinal direction of the optical fibers aligned with the reinforcement fibers of the embedding plies. The left PCFBG is oriented with $\alpha=0^{\circ}$, whilst the right sensor is oriented with $\alpha=90^{\circ}$. The 2 PCFBGs are stripped over a few centimeters at the grating location. The axis system $\left(1^{\mathrm{h}}, 2^{\mathrm{h}}, 3^{\mathrm{h}}\right)$ represents the coordinate system within the host composite material. The black double arrows indicate the directions of the carbon reinforcement fibers in the plies.

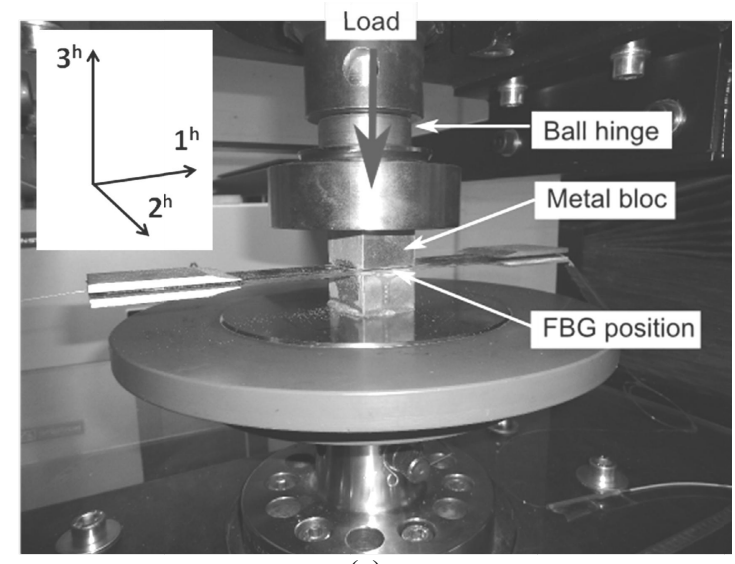

(a)

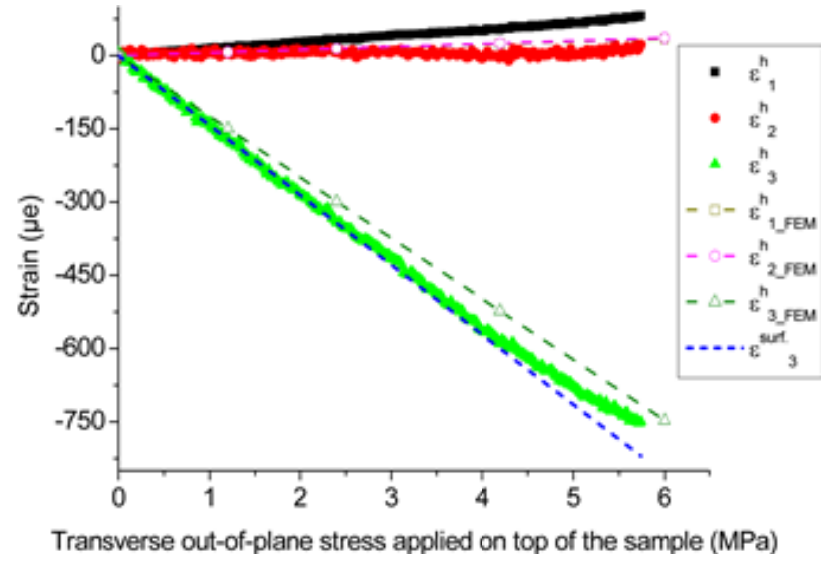

(b)

Figure 4: (a) Experimental set-up used for transverse out-of-the plane loading of a CFRP coupon. The CFRP sample is compressed between two metal blocs along the direction through the thickness. The coordinate system $\left(1^{\mathrm{h}}, 2^{\mathrm{h}}, 3^{\mathrm{h}}\right)$ of the composite sample is indicated. (b) Measured strains in $[90 / 0]_{2 \mathrm{~s}}$ and $[0 / 90]_{2 \mathrm{~s}}$ laminates as a function of the applied transverse out-of-plane stress. $\varepsilon_{1}^{h}, \varepsilon_{2}^{h}$ and $\varepsilon_{3}^{h}$ have been calculated with Equation (4). $\varepsilon_{1_{-} F E M}^{h}, \varepsilon_{2_{-} F E M}^{h}$ and $\varepsilon_{3_{-} F E M}^{h}$ are the results of our finite element simulations. $\varepsilon_{3}^{\text {surf }}$ is the strain in the direction through the thickness, calculated from the load cell reading and from the material parameters of the laminate.

\section{Composite cure monitoring and residual strain quantification}

In earlier work we have shown that it is possible to efficiently integrate Butterfly PCFBG based sensors in carbon fiber reinforced composite (CFRP) material to monitor its manufacturing process. The sensors allow assessing the onset of polymerization and quantifying the internal residual strain in the composite material that was created as a result of the vacuum bagging autoclave process [24]-[26].

Figure 5(a) shows a scheme of the CFRP specimen in which the Butterfly PCFBG was integrated and the evolution of the Bragg wavelength separation $\Delta \lambda$ during the cure cycle. The temperature variation recorded by thermocouple TC6 placed in close vicinity to the PCFBG is shown as well. Figure 5(b) shows a first drop of $\Delta \lambda$ (part B), which corresponds to the polymerization of the sample. The cooling phase (part D) features a large decrease of $\Delta \lambda$ associated with the buildup of residual strains during the consolidation phase. Since the phase modal birefringence of the Butterfly PCF is 
inherently insensitive to temperature, the changes in $\Delta \lambda$ are due to thermally induced transverse strain resulting from the changing strain state in the CFRP material as it cures.

In part $\mathrm{B}, \Delta \lambda$ decreases with $22 \mathrm{pm}$ which corresponds to a compressive transverse strain of about $-100 \mu$ strain. In region $\mathrm{C}$, the sensor signal remains constant meaning that the PCFBG sensor measures no transverse strain. One can therefore reasonably assume that the cure reaction has been completed and that the composite material is formed. The cooling down to room temperature (part D) is associated with a large decrease of $\Delta \lambda$ and thus with the development of substantial transverse residual strain of about $-1100 \mu$ strain in the composite material. The conversion from change in $\Delta \lambda$ to actual transverse strain values is obtained using data from previous sensor calibrations, as explained in [26].

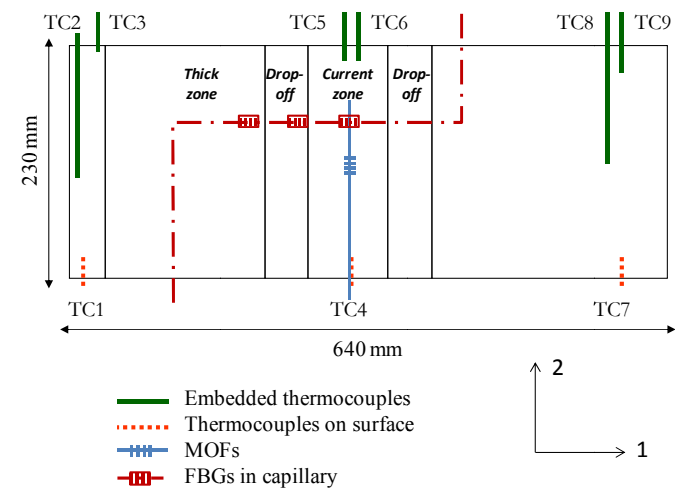

(a)

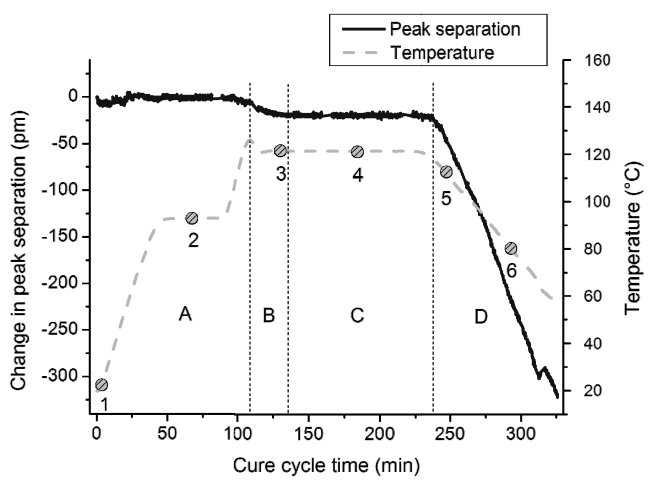

(b)

Figure 5: (a) Schematic of the optical fiber network embedded in the composite specimen. The coordinate system of the laminate is indicated. TC stands for thermocouple. (b) Changes in the temperature and in the peak separation $\Delta \lambda$ during the entire cure cycle. The temperature profile is recorded with thermocouple TC6 (Adapted from [26]).

\section{SHEAR STRAIN AND DISBOND MONITORING WITH THE BUTTERFLY PCF}

We have also shown that it is possible to measure shear strain in glued joints with a butterfly PCF that is embedded such that the transverse strain sensing axes of the PCF are aligned with the directions of principal stress in a shear loaded single lap adhesive joint (SLJ) [27]. The scheme of the embedded sensor and the measured change of the Bragg wavelength separation with applied load are shown in Figure 6. We obtained a shear stress sensitivity of about $60 \mathrm{pm} / \mathrm{MPa}$, which corresponds to a shear strain sensitivity of $0.01 \mathrm{pm} / \mu \varepsilon$. Compared to conventional birefringent fibers and as discussed in [27], our dedicated PCF design has a fourfold larger sensitivity. These results also show that by changing the angular orientation of the butterfly PCFBG sensor it can be used for either shear strain sensing or for transverse strain sensing, which provides opportunities for multi-axial strain sensing with the same type of sensor [28].

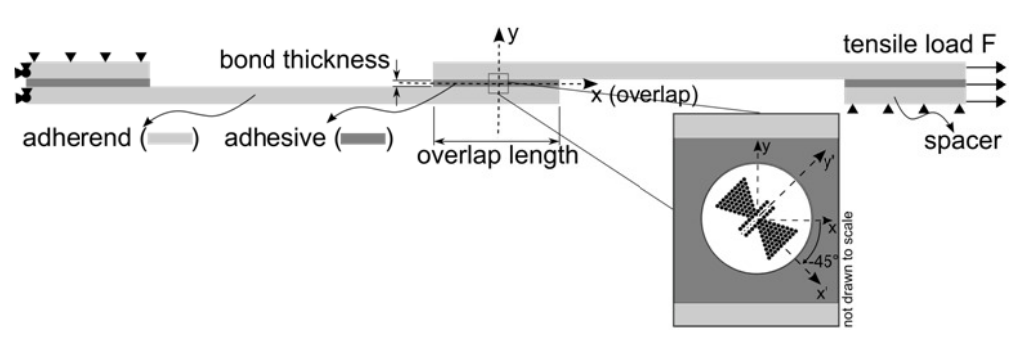

(a)

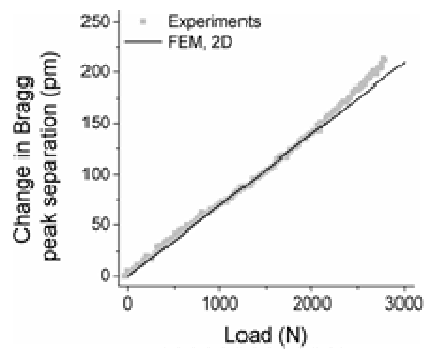

(b)

Figure 6: (a) Configuration of the tested and modeled SLJ with an optical fiber embedded in the center of the adhesive layer.

(b) The Bragg peak separation increases due to tensile loading with a sensor response of $67.4 \mathrm{pm} / \mathrm{kN}$. Results from $2 \mathrm{D}$

FEM modeling of the SLJ are in very good agreement with the experimental results (Adapted from [27]).

The combination of multiple shear stress sensors can provide insight in the existence and propagation of disbonds in SLJs. To demonstrate that ability, we have embedded three butterfly PCFBG shear stress sensors in a SLJ at the specific locations where the magnitude of the peel stress is minimal (predicted with Goland-Reissner theory): Sensor 1 and 3 near 
the edges and sensor 2 in the middle. The SLJs were made of two aluminum laps bonded by a two-component epoxy adhesive (Huntsman Araldite ${ }^{2} 2015, \mathrm{E}=1850 \mathrm{MPa}, \mathrm{G}=650 \mathrm{MPa}$ and $v=0.42$ ). We subjected the sample to a cyclic tensile loading test the response of the 3 sensors and their (collective) ability to detect disbond initiation and propagation by monitoring their response throughout the fatigue test.

The cyclic load was applied at a frequency of $2 \mathrm{~Hz}$, following a haversine trend and using a displacement controlled tensile load. The optimal maximum load was at $45 \%$ of the failure load $\left(\mathrm{F}_{\max }=2.6 \mathrm{kN}\right)$, leading to a cycle time of several tens of thousands of cycles before the joint broke (Setup is shown in Figure 7(a)). At regular intervals we calibrated the response of the PCFBG sensors using a commercially available FBG interrogation unit (FBG-scan 608). The evolution of the sensor's response throughout the complete cyclic test is a measure of the redistribution of shear stress in the bond layer, which provides information on the initiation and propagation of cracks or disbonds. Up to almost 10000 cycles the response of sensor 2 remains nearly constant with a relative change of less than $10 \%$. Afterwards the response increases to a maximum of almost 19400 cycles $(+313 \%)$ and decreases again before sample failure. The responses of both sensor 1 and sensor 3 strongly increase during the first half of the test. After 8000 cycles for sensor $3(+45 \%)$ and 10000 cycles for sensor $1(+37 \%)$, respectively, this trend changes and their responses decrease to nearly zero. By reconstructing the response of the sensors through 2D FE analysis, we could derive the length of the cracks on both sides of the SLJ (Figure 7 (b)). As can be noticed in Figure 7 (c) the reconstructed model (empty scatter points) is in very good agreement with the experiments (solid scatter points).

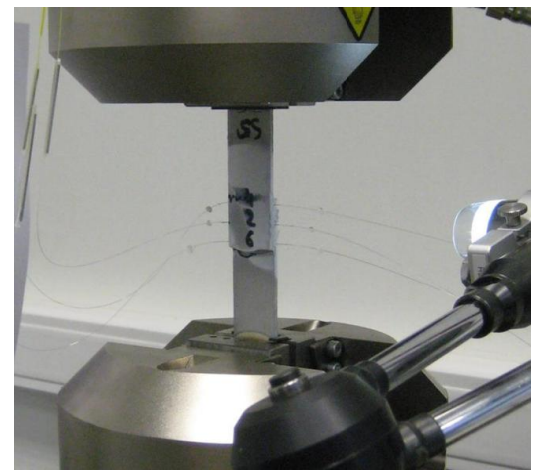

(a)

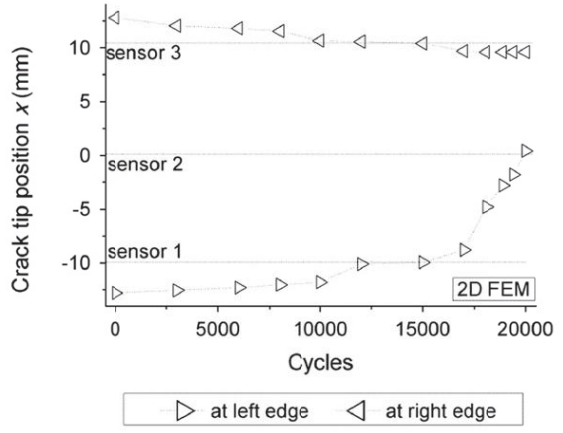

(b)

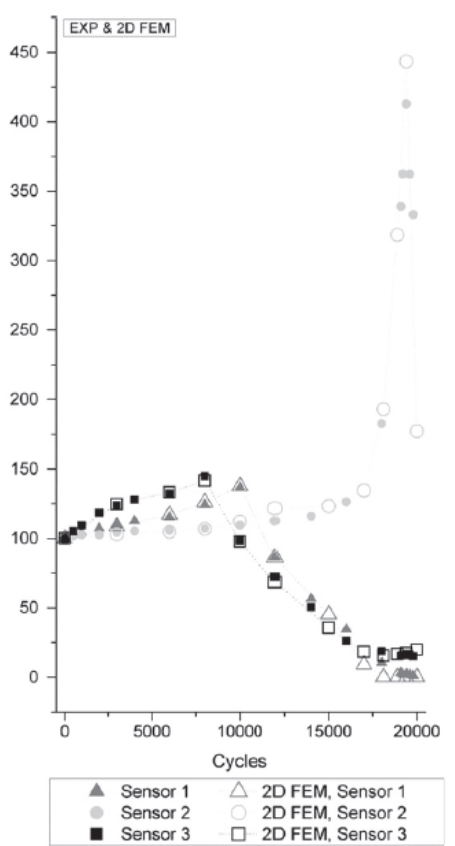

(c)

Figure 7: (a) Photograph of the sample placed in the tensile loading test setup. (b) Disbonding length on both sides of the adhesive during the cyclic test, reconstructed via FE simulations. (c) Evolution recorded during the low frequency cycles in the fatigue test of the percentage change with respect to the initial sensor response. (Adapted from [29]).

Our results demonstrate that by monitoring the response of shear stress fiber optic sensors integrated in a SLJ, we can continuously assess the shear stress distribution and hence detect the initiation and propagation of disbonds. Moreover, we find that when a disbond is present with a length of $1 \%$ of the initial overlap length, the sensor response can already change by $10 \%$. In real applications, however, it would be impractical to expose the structure to a calibrated load in order to determine the sensor sensitivity. The configuration discussed previously can nevertheless still be used when the central sensor (sensor 2) is used as a reference signal for the signals of sensors 1 and 3. As long as the bond line remains intact, the ratios $R_{12}$ and $R_{32}$, defined as

$$
R_{12}=\frac{d\left(\Delta \lambda_{\text {sensor } 1}\right)}{d\left(\Delta \lambda_{\text {sensor } 2)}\right)} ; R_{32}=\frac{d\left(\Delta \lambda_{\text {sensor } 3}\right)}{d\left(\Delta \lambda_{\text {sensor } 2}\right)}
$$


will nearly remain constant, independently of the loading level. However, once a significant disbond starts to appear at one or both sides, these ratios will change. As derived in [29], with the presented sensor configuration based on three butterfly PCFBG shear stress sensors, the minimum length of a detectable disbond is $100 \mu \mathrm{m}$, while the exact loading level does not need to be known (but should be above $17 \%$ of the failure load). These results show that the initiation and growth of disbonds in an adhesive joint can be quantitatively evaluated in a self-referencing manner, provided that one uses at least three shear stress PCFBG sensors.

\section{CONCLUSION}

We have introduced the principles of operation of a fiber Bragg grating based sensor inscribed in a highly birefringent photonic crystal fiber that has been designed so as to feature an enhanced sensitivity to transverse strain whilst being almost insensitive to temperature changes. We have illustrated how this sensor can be used in various applications that may support structural health monitoring, in particular three-dimensional strain measurements inside composite materials, cure process monitoring of and residual strain quantification in composite materials and shear stress and disbond monitoring in adhesive joints.

\section{ACKNOWLEDGEMENTS}

The authors would like to acknowledge financial support from the Agency for Innovation by Science and Technology (IWT) for funding this research with the SBO Project grant 120024 'Self-Sensing Composites'. The authors also acknowledge the Research Foundation - Flanders (FWO), the Methusalem and Hercules Foundations Flanders, and the COST action TD1001.

\section{REFERENCES}

[1] O. Frazao, J.L. Santos, F. M. Araujo, L.A. Ferreira, "Optical sensing with photonic crystal fibers," Laser \& Photonics Reviews 2(6), 449-459 (2008).

[2] J. Canning, "Properties of Specialist Fibres and Bragg Gratings for Optical Fiber Sensors," Journal of Sensors 2012, 598178 (2012).

[3] A.M.R. Pinto, M. Lopez-Amo, "Photonic Crystal Fibers for Sensing Applications," Journal of Sensors 2009, 871580 (2009).

[4] O. Frazão, J. P. Carvalho, L. A. Ferreira, F. M. Araújo, J. L. Santos, "Discrimination of strain and temperature using Bragg gratings in microstructured and standard optical fibres," Measurement Science and Technology 16, 2109-2113 (2005).

[5] H.R. Sørensen, J. Canning, J. Lægsgaard, K. Hansen, "Control of the wavelength dependent thermooptic coefficients in structured fibres," Optics Express, 14(14), 6428-6433 (2006).

[6] C. Chen, A. Laronche, G. Bouwmans, L. Bigot, Y. Quiquempois, J. Albert, "Sensitivity of photonic crystal fiber modes to temperature, strain and external refractive index," Optics Express, 16(13), 9645-9653 (2008).

[7] Y. Wang, H. Bartelt, W. Ecke, R. Willsch, J. Kobelke, M. Kautz, S. Brueckner, M. Rothhardt, "Sensing properties of fiber Bragg gratings in small-core Ge-doped photonic crystal fibers," Optics Communications 282, 1129-1134 (2009).

[8] T. Geernaert, G. Luyckx, E. Voet, T. Nasilowski, K. Chah, M. Becker, H. Bartelt, W. Urbanczyk, J. Wojcik, W. De Waele, J. Degrieck, H. Terryn, F. Berghmans, H. Thienpont, "Transversal Load Sensing With Fiber Bragg Gratings in Microstructured Optical Fibers," IEEE Photonics Technology Letters, 21(1), 6-8 (2009).

[9] G. Luyckx, E. Voet, T. Geernaert, K. Chah, T. Nasilowski, W. De Waele, W. Van Paepegem, M. Becker, H. Bartelt, W. Urbanczyk, J. Wojcik, J. Degrieck, F. Berghmans, H. Thienpont, "Response of FBGs in Microstructured and Bow Tie Fibers Embedded in Laminated Composite," IEEE Photonics Technology Letters 21(18), 1290-1292 (2009).

[10] J.-H. Zhang, N.-L. Liu, Y. Wang, L.-L. Ji, P.-X. Lu, "Dual-Peak Bragg Gratings Inscribed in an All-Solid Photonic Bandgap Fiber for Sensing Applications," Chinese Physics Letters 29(7), 074205-1-4 (2012).

[11]L.A. Fernandes, M. Becker, O. Frazão, K. Schuster, J. Kobelke, M. Rothhardt, H. Bartelt, J.L. Santos, P.V.S. Marques, "Temperature and Strain Sensing With Femtosecond Laser Written Bragg Gratings in Defect and Nondefect Suspended-Silica-Core Fibers," IEEE Photonics Technology Letters 24(7), 554-556 (2012). 
[12] L. Jin, Z. Wang, Q. Fang, Y. Liu, B. Liu, G. Kai, X. Dong, "Spectral characteristics and bend response of Bragg gratings inscribed in all-solid bandgap fibers," Optics Express 15(23), 15555-15565 (2007).

[13] L. Jin, W. Jin, J. Ju, "Directional Bend Sensing With a CO2-Laser-Inscribed Long Period Grating in a Photonic Crystal Fiber," Journal of Lightwave Technology 27(21), 4884-4891 (2009).

[14]F. Berghmans, T. Geernaert, T. Baghdasaryan, H. Thienpont, "Challenges in the fabrication of fibre Bragg gratings in silica and polymer microstructured optical fibres," Laser \& Photonics Reviews 8(1), 27-52 (2014).

[15] T. Martynkien, G. Statkiewicz-Barabach, J. Olszewski, J. Wojcik, P. Mergo, T. Geernaert, C. Sonnenfeld, A. Anuszkiewicz, M. K. Szczurowski, K. Tarnowski, M. Makara, K. Skorupski, J. Klimek, K. Poturaj, W. Urbanczyk, T. Nasilowski, F. Berghmans, H. Thienpont, "Highly birefringent microstructured fibers with enhanced sensitivity to hydrostatic pressure," Optics Express 18(14), 15113-15121 (2010).

[16] www.comsol.com

[17]F. Berghmans, T. Geernaert, M. Napierała, T. Baghdasaryan, C. Sonnenfeld, S. Sulejmani, T. Nasiłowski, P. Mergo, T. Martynkien, W. Urbanzcyk, E. Beres-Pawlik, H. Thienpont, "Applying optical design methods to the development of application specific photonic crystal fibres," Proceedings of SPIE 8550, 85500B (2012).

[18] S. Sulejmani, C. Sonnenfeld, T. Geernaert, P. Mergo, M. Makara, K. Poturaj, K. Skorupski, T. Martynkien, G. Statkiewicz-Barabach, J. Olzeswki, W. Urbanczyk, C. Caucheteur, K. Chah, P. Mégret, H. Terryn, J. Van Roosbroeck, F. Berghmans, H. Thienpont, "Control Over the Pressure Sensitivity of Bragg Grating-Based Sensors in Highly Birefringent Microstructured Optical Fibers," IEEE Photonics Technology Letters 24(6), 527-529 (2012).

[19] C. Sonnenfeld, S. Sulejmani, T. Geernaert, S. Eve, N. Lammens, G. Luyckx, E. Voet, J. Degrieck W. Urbanczyk, P. Mergo, M. Becker, H. Bartelt, F. Berghmans, H. Thienpont, "Microstructured Optical Fiber Sensors Embedded in a Laminate Composite for Smart Material Applications," Sensors 11, 2566-2579 (2011).

[20] G. Luyckx, E. Voet, W. D. Waele, J. Degrieck, "Multi-axial strain transfer from laminated CFRP composites to embedded Bragg sensor: I. Parametric study," Smart Materials and Structures 19(10), 105017 (2010).

[21]E. Voet, G. Luyckx, W. De Waele, J. Degrieck, "Multi-axial strain transfer from laminated CFRP composites to embedded Bragg sensor: II. Experimental validation," Smart Materials and Structures 19(10), 105018 (2010).

[22] C.M. Lawrence, D.V. Nelson, E. Udd, T. Bennett, "A fiber optic sensor for transverse strain measurement," Experimental Mechanics 39(3), 202-209 (1999).

[23] D. V. Nelson, A. Makino, C. M. Lawrence, J. M. Seim, W. L. Schulz, E. Udd, "Determination of the K-matrix for the multi-parameter fiber grating sensor in AD072 fibercore fiber," Proceedings of SPIE 3489, 79-85 (1998).

[24]F. Berghmans, C. Sonnenfeld, S. Sulejmani, T. Geernaert, G. Luyckx, N. Lammens, J. Degrieck, E. Voet, K. Chah, F. Collombet, W. Urbanczyk, P. Mergo, M. Becker, H. Bartelt, H. Thienpont, "Opportunities for Structural Health Monitoring of Composite Material Structures with novel Microstructured Optical Fiber Sensors," Proceedings of 9th International Workshop on Structural Health Monitoring 1, 902-909 (2013).

[25] C. Sonnenfeld, G. Luyckx, F. Collombet, Y. Grunevald, B. Douchin, L. Crouzeix, M. Torres, T. Geernaert, S. Sulejmani, S. Eve, M. Gomina, K. Chah, P. Mergo, H. Thienpont, F. Berghmans, "Embedded fibre Bragg gratings in photonic crystal fibres for cure cycle monitoring of carbon fibre reinforced polymer materials," Proceedings of SPIE 8775, 877500 (2013).

[26] C. Sonnenfeld, G. Luyckx, F. Collombet, Y-H. Grunevald, B. Douchin, L. Crouzeix, M. Torres, T. Geernaert, S. Sulejmani, K. Chah, P. Mergo, H. Thienpont, F. Berghmans, "Cure cycle monitoring of laminated carbon fiber-reinforced plastic with fiber Bragg gratings in microstructured optical fiber," Proceedings of ICCM 19, 3327-3335 (2013).

[27] S. Sulejmani, C. Sonnenfeld, T. Geernaert, G. Luyckx, D. Van Hemelrijck, P. Mergo, W. Urbanczyk, K. Chah, C. Caucheteur, P. Mégret, H. Thienpont, F. Berghmans, "Shear stress sensing with Bragg grating-based sensors in microstructured optical fibers," Optics Express 21(17), 20404-20416 (2013).

[28] S. Sulejmani, C. Sonnenfeld, T. Geernaert, D. Van Hemelrijck, G. Luyckx, P. Mergo, W. Urbanczyk, K. Chah, C. Caucheteur, P. Mégret, H. Thienpont, F. Berghmans, "Fiber Bragg grating-based shear strain sensors for adhesive bond monitoring," Proceedings of SPIE 9128, 9128-12 (2014).

[29] S. Sulejmani, C. Sonnenfeld, T. Geernaert, G. Luyckx, P. Mergo, W. Urbanczyk, K. Chah, H. Thienpont, F. Berghmans, "Disbond monitoring in adhesive joints using shear stress optical fiber sensors," Smart Mater. Struct. 23, 075006 (2014). 